\title{
More evidence against alcohol or smoking in patients with unruptured intracranial aneurysm
}

Joanna Pera, $\mathrm{MD}, \mathrm{PhD}$

Ynte M. Ruigrok, MD, $\mathrm{PhD}$

Correspondence to Dr. Ruigrok:

ij.m.ruigrok@umcutrecht.nl

Neurology ${ }^{\circledR} 2015 ; 84: 442-443$
Intracranial aneurysms are common, with a prevalence of $3 \%$ in the general population. ${ }^{1}$ The growing availability of brain imaging has increased the number of incidentally discovered aneurysms. Rupture of intracranial aneurysms and the resulting subarachnoid hemorrhage (SAH) has a high case fatality and morbidity, and occurs at a relatively young age compared with other types of stroke. ${ }^{2}$ As yet, we do not have a medical treatment to prevent intracranial aneurysm formation and its subsequent rupture. Our only preventive treatments for existing aneurysms consist of invasive surgery or endovascular treatment, each with inherent risk of complications including death or permanent disability. ${ }^{3}$ The risks of treatment have to be balanced carefully against the risk of rupture. ${ }^{4}$ Consequently, an important recent focus of aneurysm research has been the identification of risk factors of aneurysmal rupture. Recently, a meta-analysis of 6 prospective cohort studies showed that prognostic factors for aneurysm rupture include age, hypertension, history of SAH, aneurysm size, aneurysm location, and Finnish or Japanese descent. ${ }^{5}$ Other factors might also predict aneurysm rupture, including smoking $^{6}$ and family history of SAH.

In this issue of Neurology ${ }^{\circledR}$, Juvela and Lehto ${ }^{8}$ now focus on all-cause mortality and risk factors for death in patients with unruptured intracranial aneurysms (UIAs) about which little is known. The authors report on a unique long-term follow-up study in 142 patients with 181 UIAs diagnosed between 1956 and 1978 and followed until 2011-2012, resulting in 3,530 person-years of follow-up. During follow-up, 113 of the 142 patients (80\%) had died. Of the 113 who had died, 27 patients (24\%) died of aneurysm rupture or of other aneurysm-related causes while the remaining 86 patients $(76 \%)$ died of other causes that were unrelated to their aneurysms. Independent risk factors for all-cause death were patient age, male sex, heavy alcohol use, and cigarette smoking. History of hypertension, family history of SAH, and diameter of the UIA were associated with allcause death only in the univariable analysis. These risk factors were not independent.
An important strength of this study is the large cohort of patients with UIAs for which a complete and long-term follow-up is available, allowing for an ideal cohort of patients to study all-cause mortality and risk factors for death. However, the study also has limitations. First, the patient cohort may not be representative of the general population of patients with UIAs. In this study, the majority of the patients (131 of 142 patients, 92.2\%) had multiple aneurysms and another aneurysm had already ruptured and been treated. Because a history of $\mathrm{SAH}^{5}$ and possibly also multiple aneurysms ${ }^{9}$ are risk factors for aneurysm rupture, this population is likely to be at a higher risk of aneurysmal rupture and consequently also at a higher risk of death due to aneurysm-related problems. Second, all patients were diagnosed between 1956 and 1978 and many died of cardiovascular events. Secondary prevention strategies in cardiovascular disease have improved dramatically in the intervening years. Thus, the results that were seen in the patients in this study may not apply to patients that are currently identified with UIAs. Lastly, based on their findings, the authors conclude that in patients with UIA, alcohol consumption and cigarette smoking are modifiable risk factors for all causes of death and that these risk factors should be taken into account when treatment is considered. However, we already know that smoking and heavy alcohol consumption are important risk factors for mortality, including mortality from cardiovascular events, in the general population. ${ }^{10}$ From this study, we do not learn whether patients with UIA are at increased risk of death due to alcohol consumption and cigarette smoking as compared with the general population, because no independent reference cohort is used for comparison to the patients with UIAs. We already knew that we should counsel patients with UIA about lifestyle. The current findings do not tell us whether this counseling should be intensified.

Nonetheless, despite these limitations, this study analyzing a unique long-term cohort of patients with UIAs provides important further insights in long-term 
outcome with an emphasis on the causes and risk factors of death of patients with UIAs.

\section{STUDY FUNDING}

No targeted funding reported.

\section{DISCLOSURE}

The authors report no disclosures relevant to the manuscript. Go to Neurology.org for full disclosures.

\section{REFERENCES}

1. Vlak MH, Algra A, Brandenburg R, Rinkel GJ. Prevalence of unruptured intracranial aneurysms, with emphasis on sex, age, co-morbidity, country, and time period: a systematic review and meta-analysis. Lancet Neurol 2011;10: 626-636.

2. Rinkel GJ, Algra A. Long-term outcomes of patients with aneurysmal subarachnoid haemorrhage. Lancet Neurol 2011;10:349-356.

3. McDonald JS, McDonald RJ, Fan J, Kallmes DF, Lanzino G, Cloft HJ. Comparative effectiveness of ruptured cerebral aneurysm therapies: propensity score analysis of clipping versus coiling. AJNR Am J Neuroradiol 2014; 35:164-169.
4. Etminan N, Beseoglu K, Barrow DL, et al. Multidisciplinary consensus on assessment of unruptured intracranial aneurysms: proposal of an international research group. Stroke 2014;45:1523-1530.

5. Greving JP, Wermer MJ, Brown RD Jr, et al. Development of the PHASES score for prediction of risk of rupture of intracranial aneurysms: a pooled analysis of six prospective cohort studies. Lancet Neurol 2014;13:59-66.

6. Juvela S, Poussa K, Lehto H, Porras M. Natural history of unruptured intracranial aneurysms: a long-term follow-up study. Stroke 2013;44:2414-2421.

7. Broderick JP, Brown RD Jr, Sauerbeck L, et al; FIA Study Investigators. Greater rupture risk for familial as compared to sporadic unruptured intracranial aneurysms. Stroke 2009;40:1952-1957.

8. Juvela S, Lehto H. Risk factors for all-cause death after diagnosis of unruptured intracranial aneurysms. Neurology 2015;84:456-463.

9. Sonobe M, Yamazaki T, Yonekura M, Kikuchi H. Small unruptured intracranial aneurysm verification study: SUAVe Study, Japan. Stroke 2010;41:1969-1977.

10. World Health Organization. Global Health Risks: Mortality and Burden of Disease Attributable to Selected Major Risks. Geneva: World Health Organization; 2009. 